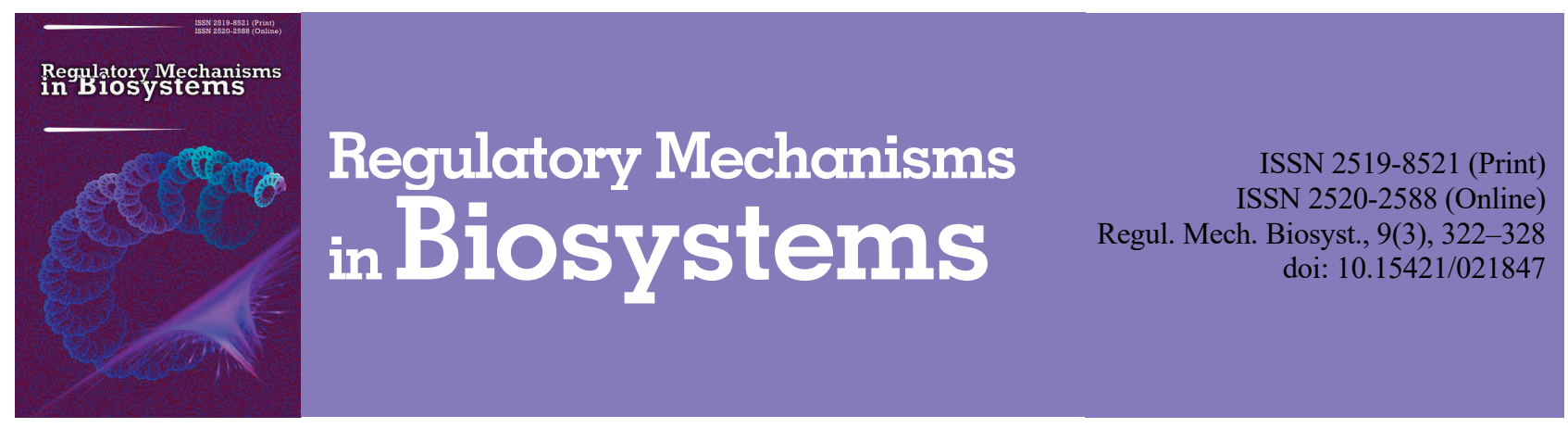

\title{
Fibrin-blood clot as an initial stage of formation of bone regeneration after a bone fracture
}

\author{
O. K. Popsuishapka*, N. O. Ashukina**, V. O. Litvishko***, \\ V. V. Grigorjev****, O. O. Pidgaiska**, K. O. Popsuishapka** \\ *Kharkiv Medical Academy for Postgraduate Education of the Ministry of Health of Ukraine, Kharkiv, Ukraine \\ **Sytenko Institute of Spine and Joint Pathology National Academy of Medical Sciences of Ukraine, Kharkiv, Ukraine \\ ***Kononenko Chuguyev Central District Hospital», Chuguyev, Ukraine \\ ****Cherkasy City Accident and Emergency Hospital No 3, Cherkasy, Ukraine
}

Article info

Received 19.05.2018 Received in revised form 07.07.2018 Accepted 10.07.2018

Kharkiv Medical Academy of Postgraduate Education Amosov st., 58,

Kharkiv, 61176, Ukraine.

Tel.: +38-067-933-51-71 E-mail:

alexeycorn@gmail.com

Popsuishapka, O. K., Ashukina, N. O., Litvishko, V. O., Grigorjev, V. V., Pidgaiska, O. O., \& Popsuishapka, K. O. (2018). Fibrin-blood clot as an initial stage of formation of bone regeneration after a bone fracture. Regulatory Mechanisms in Biosystems, 9(3), 322-328. doi:10.15421/021847

This study focuses on mechanisms which regulate the process of fracture healing. We studied the form and position of the fibrin-blood clots (FBC) in the zone near the fragments among patients with limb fractures: macroscopically (during open surgical operations to reposition bone fragments) and using sonography. We conducted a histological and immunohistochemical analysis of biopsy material obtained from the zone around the fracture during surgical procedures on 16 patients in 1-18 days after the fracture. We determined that the density of FBC and their form depends on the volume of damage to the periosteum-muscular fascia and the extent of the shift of fragments. In most cases, with a closed fracture, fibrin-blood clots had a spindle-shaped form. Fibrin along the periphery and in the zone between the fragments has a dense structure, and becomes cellular in central zones. The cells surrounded by fibrin partitions contain blood cells and serum. In many places, fibrin partitions had a one-direction orientation position, and the cells were oval-elongated, which indicated hydrostatic pressure in them. Proliferation of mesenchymal cells began in the vital tissues around the $\mathrm{FBC}$, then during reproduction they pentrated to fibrin. Lengthwise axis of the cells was parallel to fibrin partitions. The bone trabeculae which form on the third week after fracture repeated the orientation of the fibrin partitions. It was determined that the vascular endothelial growth factor (VGEF) concentrates in fibrin and remains in it over the first week after the fracture, later it was found in endotheliocytes, fibroblasts and osteoblasts. The process of filling of the cells with around-fracture FBC lasted 1218 days and during this period, their osteogenic differentiation occurred. Such tempi and orientation of the process is caused by fibrin with a concentration of growth factors in it. Using the results of the study, it could be assumed that the main conditions for osteogenic differentiation of cells are high concentration of VGEF in the fibrin, which initiates neoangiogenesis and internal tension of fibrin partitions. The formation of structured FBC around the ends of the fragments, which contain VGEF should be considered an initial stage of the process of forming of bone regeneration.

Keywords: structure of fibrin; vascular endothelial growth factor; reproduction of mesenchymal cells; orientation of mesenchymal cells; orientation of bone trabeculae.

\section{Introduction}

In traumatology, there has recently been a tendency towards surgical treatment of fractures of limb bones. This could be related to the intense impact of the powerful industry of preparations for advanced osteosynthesis. At the same time, it would be wrong to state that technically advanced devices and methods of their use are in harmony with formation of the regenerated bone. Most of them are designed for an open cutaneous approach to the fractured bone, which increase the risk of disrupting the regeneration process and development of pus-necrotic infectious complications.

In cases of multiple closed fractures, especially if the fragments are shifted over an insignificant distance, there are two possible methods of treatment - closed reduction of the fragments and fixation by an external device or open reduction and stabilization using a internal fixating device. The question which of the mentioned variants causes fewer complications and has a higher clinical efficiency is still to be solved. Some researchers present different frequencies of nonunion of shaft fractures after internal osteosynthesis (Gaebler et al., 2001). According to the results of an independent study, it is significantly higher (Antonova et al., 2013). In the conditions of intense development of the industry of preparations for healing fractures, the selection of the approach for fixating the fragments is significantly affected by factors of an economic-commercial character, which can sometimes infringe biological regulations.

Understanding of the pattern of bone healing is necessary for choosing an adequate method of restoration of bone fragments, which would cause the least damage to the natural processes of bone regeneration. This requires knowledge and understanding of the mechanisms that organize this process. Currently the data is still insufficient.

Recent scientific publications present the following stages in the process of bone regeneration: I - inflammation (1-7 days), II - formation of soft callus (2-3 weeks), III - formation of hard callus (34 months), IV - remodeling of the new formed bone (5 months and 
longer) (Sfieretal., 2005; Ito \& Perren, 2007). In our opinion, the basis of the division of regeneration process into stages should be determined by the mechanisms which regulate the natural process. Maximum activity occurs during the initial stages of the processes and phenomena which take place. Researchers have different opinions on this topic. Some focus on the inflammatory as the factor which initiates the process (Marsell \& Einhom, 2012), other focus on the role of hematoma (Schell et al., 2017) or on the process of formation of the fibrin-collagen carcass (Popsuishapka et al., 2013; Echeverri et al., 2015; Wang et al., 2016).

Significant progress in the understanding of the process of bone regeneration was made by the studies of clinical use of fibrin enriched with thrombocytes (platelet-rich fibrin, PRF) in dental implantology (Ehrenfest et al., 2010; Kobayashi et al., 2015; Aydemir Turka et al., 2016; Bastami \& Khojasteh, 2016). As we know, neoangenesis and differentiation of osteoblasts are regulated by biologically active factors: the vascular endothelial growth factor (VEGF) (Ramasamy et al., 2016; Hu \& Olsen, 2016), transforming growth factor (TGF) (van Meeteren et al., 2011), bone morphogenetic proteins (BMP) (MorenoMiralles et al., 2009), platelet-derived growth factor (PDGF) (Raica et al., 2010). The abovementioned factors (except BMP) are contained in the granules of platelets and some other white blood cells which possibly become released from fibrinogen in the process of forming fibrin. The discovery that mesenchymal cells can differentiate to osteoblasts if the regeneration object has vessels is highly significant (Maes et al., 2010; Marsell \& Einhorn, 2011; Hankenson et al., 2011; Sivaraj \& Adams, 2016; Ramasamy et al., 2016). Therefore, special attention should be focused on the role of VEGF in the process of osteoregeneration (Dong et al., 2016; Hu \& Olsen, 2016).

In our opinion, the problem is that most of the abovementioned data were obtained mostly in theoretical-experimental studies and they do not significantly affect the existing methods of treating fractures. We mean not different commercial projects (for example, making devices for obtaining PRF), but practical suggestions regarding particular medical actions, e. g. what to do with hematoma around the fracture? Or, could anti-inflammatory preparations be prescribed in early stages after the fracture?

The objective of the study was the elaboration of the set-off mechanism and the orientation of the regenerative process after the bone fracture through the study of structural-morphological peculiarities of fibrin-blood clots in the space around the fracture, its biological activity and mechanical impacts related to the fixation regime.

\section{Materials and methods}

The study was conducted in accordance with the current ethical requirements. The protocol of the study was approved at the meeting of the Committee of Bioethics of the Sytenko Institute of Spine and Joint Pathology, Academy of Medical Science of Ukraine (protocol No 109 from 29.10.2012). A macroscopic study was conducted of the morphology of the fibrin-blood clot around the fracture among patients with diaphyseal fractures of long bones. During open reduction of the fragments for 45 patients with closed diaphyseal fracture, we assessed the form and peculiarities of positions of fibrin-blood clots in the area around the fracture.

Histological study. Biopsy material from the area around the fracture was taken from 16 patients over 1-18 days during surgical procedures and was fixated for 24 hours in $10 \%$ neutral formalin. Then the material was decalcified (if bone fragments were present) in $10 \%$ solution of formic acid, dehydrated in spirits (70\%, twice $96 \%$ ), mixture of $96 \%$ spirit with diethyl ether $(1: 1)$ and poured into celloidin. Histological sections of $5 \mu \mathrm{m}$ thickness were made using a Reichert rotary microtome (Austria), stained using hematoxylin and eosin (H\&E) and picrofuchsin solution according to Van Gieson (Van Gieson's stain). For the analysis, we used a BX63 microscope (Olympus, Japan) and the photographs were taken using a DP73 digital camera (Olympus) and "Cell Sens Dimention 1.8.1" (Olympus, 2013) software.

The zone of interest was the border between the maternal vital tissue and the fibrin-blood clot adjacent to it. We assessed the structure of fibrin, orientation of its fibrin bundles, places and periods of ap- pearance of mesenchymal cells, vessels and spatial orientation. We assessed the number of spindle-shaped cells (per 100 cells in the microscope view, lens ${ }^{\mathrm{x}} 40$, eyepiece ${ }^{\mathrm{x}} 10$ ) which orientated with the long axis from the adjacent tissues to the centre of the fibrin-blood clot along the fibrin fibres, on the 3,5,8 and 12th day after the injury. Using the IBM SPSS Statistics 20 program, we calculated the mean value $(\mathrm{x})$ and standard error (SE). The significance of the differences between the periods of monitoring was assessed using a single factor dispersal analysis.

Immunohistological study of the expression of the vascular endothelium growth factor (VEGF) in the tissues adjacent to the area of the fracture. The biopsy material was taken from 9 patients, from the tissues which surrounded the fragments, during their open reduction. The periods after the trauma: $1-5$ days -4 patients, $8-15$ days -5 . Using a lancet, we cut the fibrin-blood clot with the adjacent periosteum, muscles and adipose tissue as a single block. Biopsy material was fixated in $10 \%$ buffered formalin and processed in accordance with the generally accepted method in a Mikrom CP-120 histoprocessor, and poured into paraffin wax. Histological sections of $3 \mu \mathrm{m}$ thickness were made on the rotary microtome LeikaRM 2125, deparaffinated, and some were stained using hematoxylin and eosin. Other sections were analysed on VEGF with mouse antibody of VEGF (VG1 clone, Diagnostic Bio Systems) using the indirect peroxidase method with high-temperature exposure of En Vision (Dako) antigens. A brown colour corresponded to a positive result. The preparations were made in the laboratory of the Cherkasy Regional Oncological Dispensary (head of the dispensary - F. M. Halkin), the analysis was made in the Laboratory of Morphology of Connective Tissue of Sytenko Institute of Spine and Joint Pathology.

The results of immune-histological reaction were evaluated by the following points: 0 - reaction is absent; 1 - insignificant reaction (lower than $25 \%$ of the microscopic field), 2 - moderate (over $25 \%$, but no higher than 50\%), 3 - clear (over 50\%), 4 - maximum (100\%).

Ultrasonography of the zone around the fracture. Ultrasound analysis was conducted for 25 patients with shaft fractures of the humerus (13), tibia (4), femur (8). The periods of monitoring after the fracture: 2-6th day - 4, 7-14th - 16, 15-21st - 10. In total, 30 examinations were made, one patient was examined three times, and two patients were examined twice. We used a ToshibaAplio-500 sonograph with a linear 5-12 Hz transducer and a SLE-100 (Lithuania) mobile sonograph with $5.0-7.5 \mathrm{~Hz}$ transducer. The transducer was positioned alongside the fragment axis, exogeneity of the tissues was examined in the front and sagittal planes. We assessed the form of the external contour of the hematoma (or fibrin-blood clot) and recorded lengthwise and transversal sizes.

\section{Results}

Results of macroscopic (during surgical operation) and ultrasonographic examination. At the moment of opening the area of fracture, blood which filled the posttraumatic space had already changed its biological condition. It partly saturated adjacent tissues, and the rest of the blood had turned into a fibrin-blood clot. Taking to account these transformations, over first days after the fracture, we observed the following zones (Fig. 1):

- fibrin-blood clot zone;

- zone of serums with erythrocytes (blood fluid) (sometimes present);

- zone of muscles saturated with blood.

Depending on the magnitude of the shift of fragments and destruction of the surrounding tissues, the configuration of the fibrinblood clot was different. We observed three variants:

I - the fibrin-blood clot fully filled the space around the fracture, and was limited by detached and hardly damaged periosteum. This variant occurred when the fragments were positioned along the width at an insignificant distance, approximately $1 / 3$ of bone diameter (Fig. $2 a$ ). The periosteum with muscles under the pressure of blood deformed as a membrane, forming a spindle-shaped space filled with blood; II - fibrin-blood clot retained spindle-like shape as a periphery barrier; was limited by muscles, but had a serum with erythrocytes (blood 
fluid) in its central zone (Fig. 2, b); III - periosteum-muscular fascia was damaged, spindle-like shape was absent, fibrin-blood clots were in the form of fragments and were positioned in different places (not forming solid structure), the blood saturated the surrounding tissues in large areas, the fragments were shifted to full diameter and along the length (Fig. 2, c).

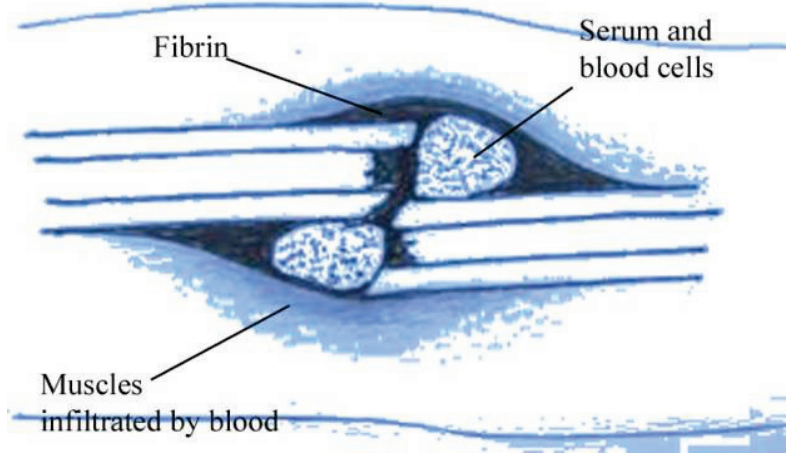

Fig. 1. Schematic image of the identified zones around the fracture

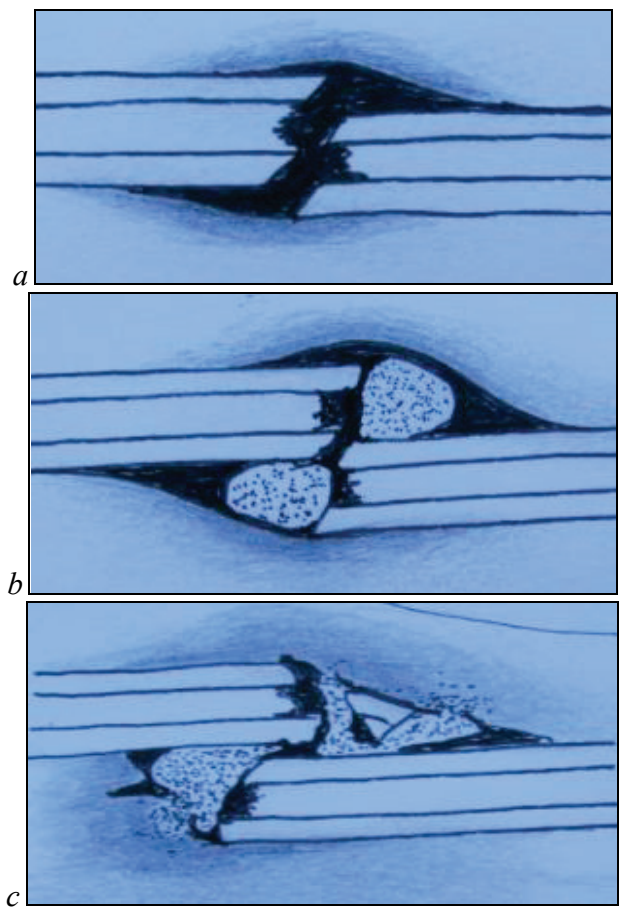

Fig. 2. Schematic illustration of the variant of the form and volume of the fibrin-blood clot in the area around the fracture after diaphyseal fracture: $a-\mathrm{I}, b-\mathrm{II}, c-\mathrm{III}$

The zone of perifocal infiltration of muscles (or other tissues) with blood depended on the extent of destruction of the periosteum barrier - the greater the disruption, the wider the distribution of broadly its infiltration. This was determined visually by change in colour of the muscles and proved by histological examination. In this zone between the muscle fibres, there were accumulations of erythrocytes. Fibrin-blood clots which filled the space around the fragments were dark-brown, had elastic consistency and were also closely connected to the surrounding tissues (bone, periosteum, myeloid and adipose tissue). Their most typical localizations were: the fractured surface of the bone, including the bone-marrow canal, then the internal surface of the detached periosteum, mostly in border zones of the spindle. If the shift of fragments was insignificant, the clot filled the space directly between the areas of fracture, as we see in the patient during a surgical operation (Fig. 3).

Results of ultrasonographic examination. During ultrasonographic examination of the fracture zone, on the 2-6th days after its appearan- ce, $72 \%$ of the patients ( 18 of 25 ) were observed to have an anechogenic zone with a bow-shaped external contour (Fig. 4) above the fragments. It indicated the presence of the hematoma around the fragments or a fresh fibrin-blood clot with no organization. To determine the level of echogenicity of freshly formed fibrin, we put a two or three day fibrin-blood clot obtained from the venous blood of patients on the transducer of the sonograph. The freshly formed clot did not resist the ultrasound and was identified by the sonograph as an anechogenic medium. Seven and more days later, at the site of the former hematoma (mostly along the peripheral part, and also directly between the fragments), a bow-shaped zone of hypoechogenicity appeared, which connected the fragments in an arc (bridge). This symptom indicated the growth of the cells in fibrin with formation of collagen. This coincided with the results of our histological studies of the clot around the fragments and other data (McNally, 2011).

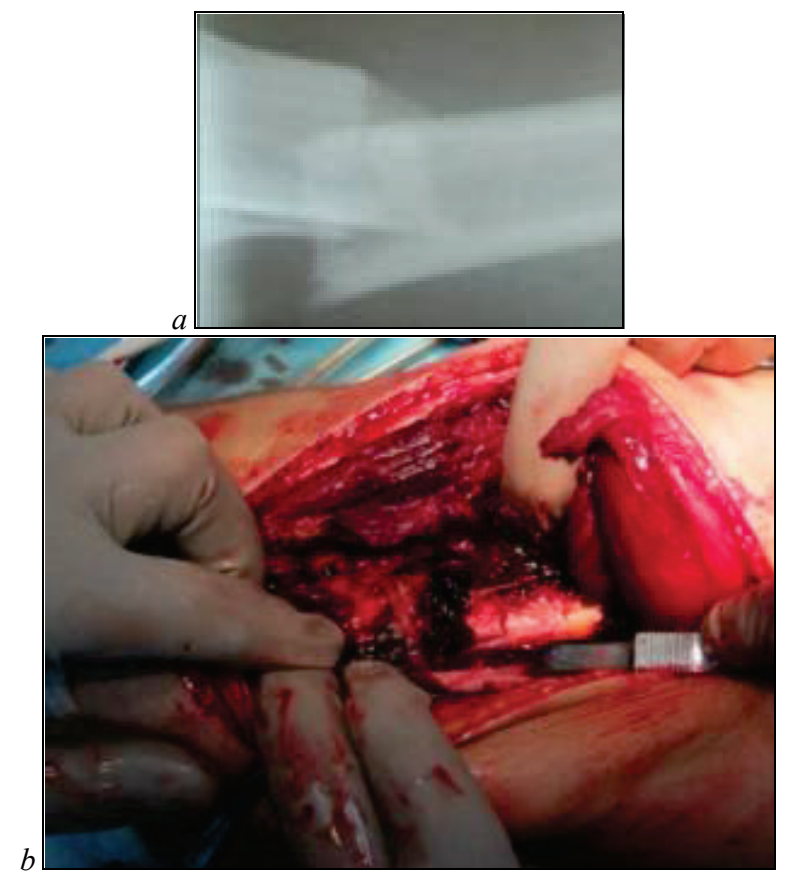

Fig. 3. Radiography of the zone of femur fracture $(a)$ and photo of positioning of fibrin-blood clot between fragments $(b)$ of patient III, 21 years, 12th day after the trauma

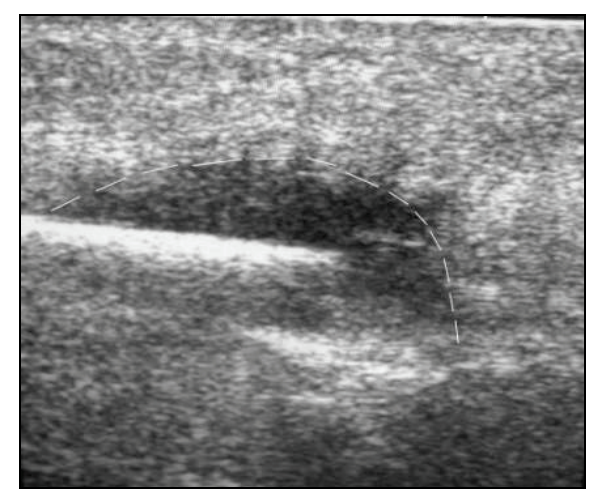

Fig. 4. Ultrasonographic image of the fracture zone of tibia: 2nd day after the trauma; anechogenic zone with bow-shaped external contour (white line)

Results of histological examinations. On histological preparation of biopsy material obtained during a surgical operation, in the first 13 days after the trauma, we found fibrin-blood clots represented by a system of fibrin membranes of different density in the zone around the fragments. In some areas, they were positioned in parallel, in others they formed a cellular structure. Cells limited by fibrin partitions were rounded or oval-elongated orientated in one-direction and filled with blood cells (mostly erythrocytes) (Fig. 5). 


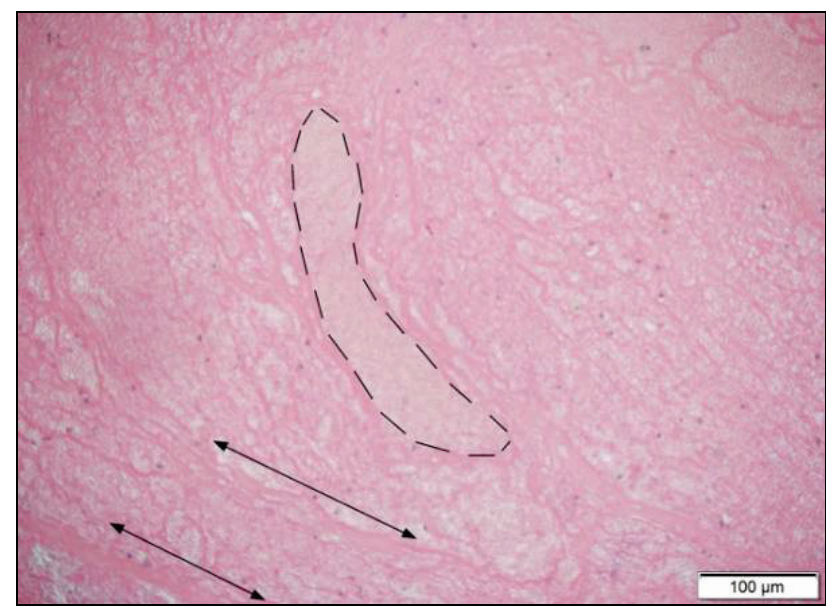

Fig. 5. Fragment of fibrin-blood clot with the zone surrounding the bone fragments, 2nd day after the fracture: the pointers indicate the orientation of the parallel fibrin membranes, dashed line -

oval-elongated cell filled with erythrocytes; bar $-50 \mu \mathrm{m}$

Starting from the $3 \mathrm{~d}$ day after the fracture, we observed capillaries developing into a fibrin-blood clot and its penetration to the blast cells of stretched shape, the long axis of which was orientated towards their direction of movement - from adjacent tissues to the center of the clot. Such cells had large hypochromic nuclei and elongated basophil cytoplasm. The density of cells (among them - insignificantly differentiated mesenchymal, fibroblasts, macrophages) was the highest on the side of damaged muscle tissue infiltrated with blood and decreased closer to the central areas of the clot (Fig. 6). Between the muscle fibers, we found bleeding in the form of accumulation of erythrocytes, mesenchymal cells, mitotic figures (2-3 in the microscope view, eyepiece ${ }^{\mathrm{x}} 10$, lens ${ }^{\mathrm{x}} 40$ ). Proliferation of insignificantly differenttiated cells was observed also in the bone-marrow canal.

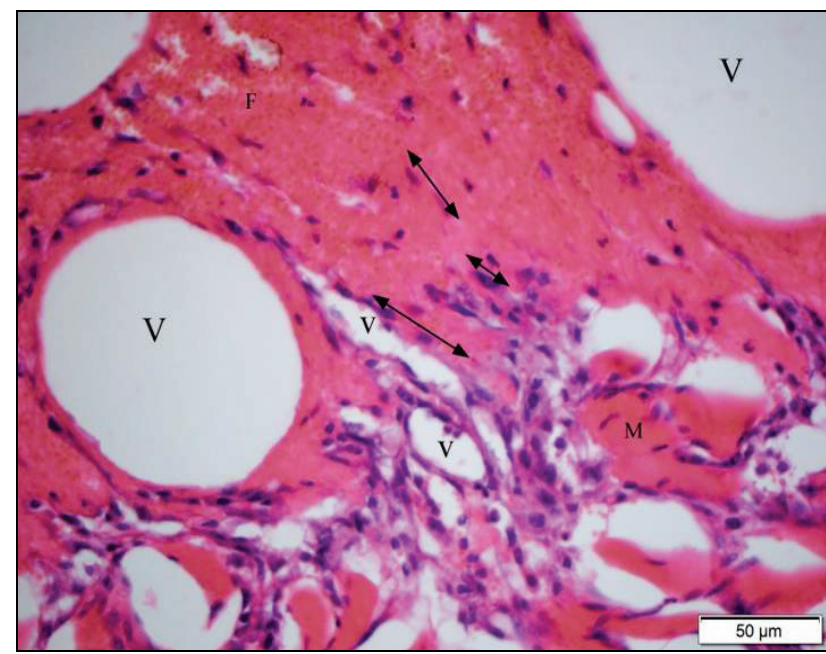

Fig. 6. Fragment of fibrin-blood clot and the muscle adjacent to it, which is infiltrated with blood, 5th day after the trauma: high cellular density at the "clot-muscle" border; the pointers indicate the orientation of growing of blood capillaries (v) and cells, 4th day after the fracture of a sheep

The longer the territory of the clot was monitored, the smaller it became due to the number of cells which penetrated to it from the surrounding tissues. Particularly, on the $3 \mathrm{~d}$ day after the fracture, only $3.7 \pm 0.66$ cells out of 100 located at the border between damaged tissues and the clot had a spindle-like shape and penetrated to it, 5 days later, their number insignificantly increased $(7.1 \pm 0.93)$, after 8 and 12 days, it had increased compared to the previous period of study by 3.08 and 1.50 times respectively $-21.9 \pm 2.0$ and $33.2 \pm 2.88(\mathrm{P}<$ 0.001) (Fig. 7). The cells formed pod-like bundles which started from the zone of their accumulation and grew into fibrin.
8-12 days after the trauma, the restructuring of the fibrin-blood clot continued: insignificantly differentiated cells penetrated to it, fibroand osteoblasts, blood capillaries which formed granular and fibroreticular tissues, and osteoids near the bone fragments. In the areas remote from the maternal damaged cells, the number of cells was lower, also we found remains of fibrin. The elongated cells on some areas of the preparation were positioned in parallel to one another and to the fibrin partitions (Fig. 8).

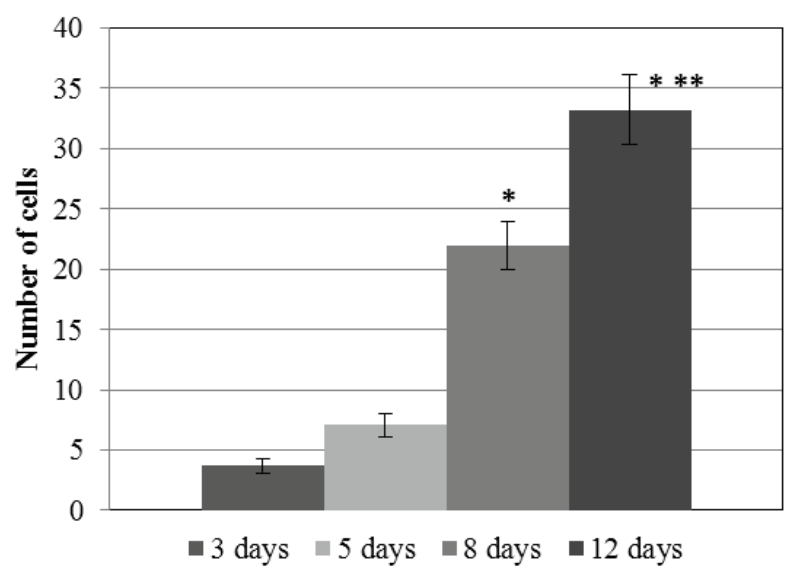

Fig. 7. Number of elongated cells which penetrated to the fibrinblood clot, depending on the period of study: the graph demonstrates the standard error of the arithmetic mean (SE Standard Error), the probability of the differences $\mathrm{P}<0.001$; * - compared to the period of 3 and 5 days, ** - compared to the period of 8 days

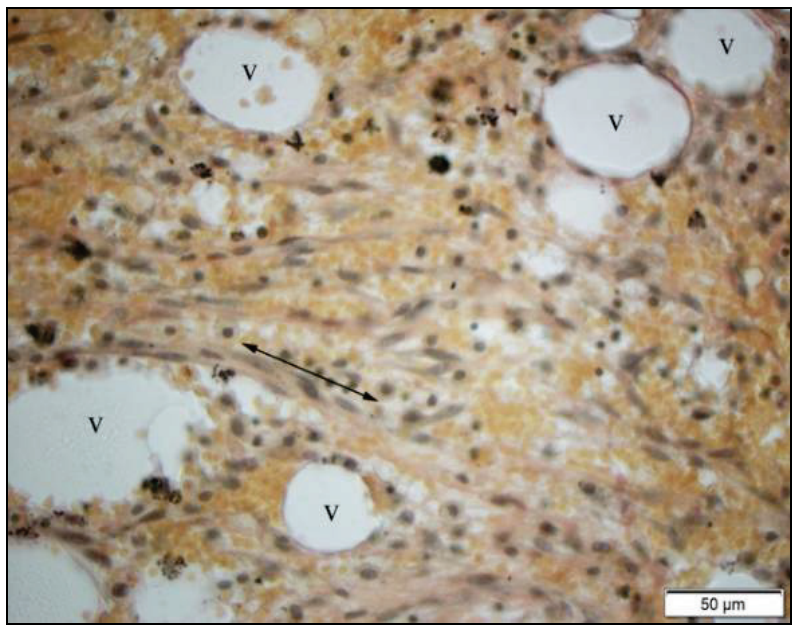

Fig. 8. Fragment of the regeneration between the fragments

9 days after the fracture of humerus: Ribbon-like proliferates of fibroblastic cells (the pointer indicates the orientation of long axis of the cells), the vessels of the capillary type (V); Van Gieson's stain

14-18 days after the fracture, at the place of the fibrin-blood clot, granular tissue formed, which had a high density of cells, vessels, areas of fibroreticular tissues of osteogenic type, and large-fiber bone tissue in the form of separate bone trabeculae. On their external surface, there were functionally active osteoblasts with large hypochromatic nuclei. Between the bone trabeculae, there was a fibroreticular tissue of osteogenic type with significant number of osteoblasts and fibroblasts. Lengthwise axis of these cells corresponded to the axis of the trabeculae (Fig. 9).

Results of immunohistological examination of VEGF in the tissues of the zone around the fragments after fractures of long bones. In the biopsy material taken on the 1-2nd day after the fracture, in the fibrin-blood clot, we found maximum reaction to VEGF (4 degrees), and in the surrounding tissues - minimum ( 0 degrees in the adipose tissue) and low (1 degree - in the muscle tissue) (Fig. 10). 


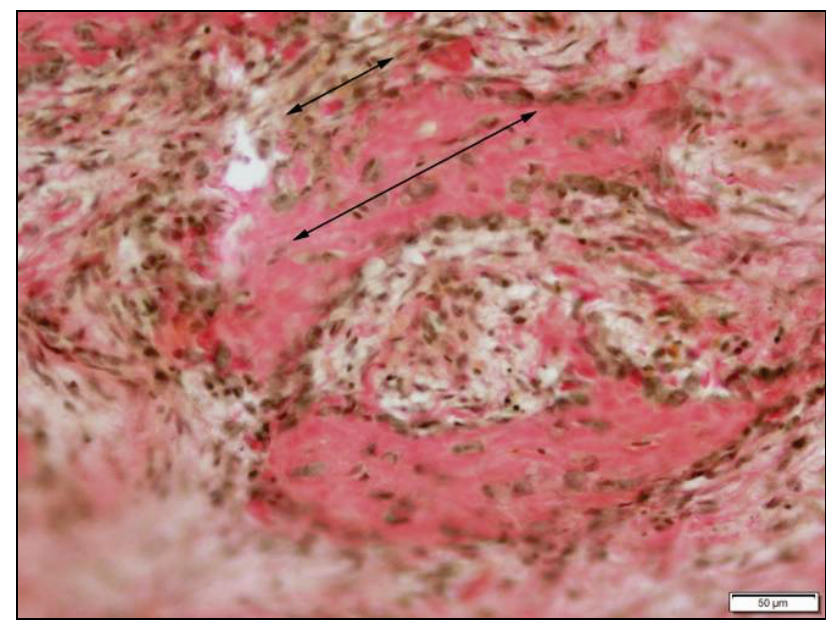

Fig. 9. Fragment of biopsy material with the surrounding area, 18th day after the fracture of tibia: the new formed bone trabeculae with active osteoblasts on the external surface; reticular fiber tissue of osteogenic type; long axis of fibroblasts is parallel to the trabeculae axis (pointers); Van Gieson's stain

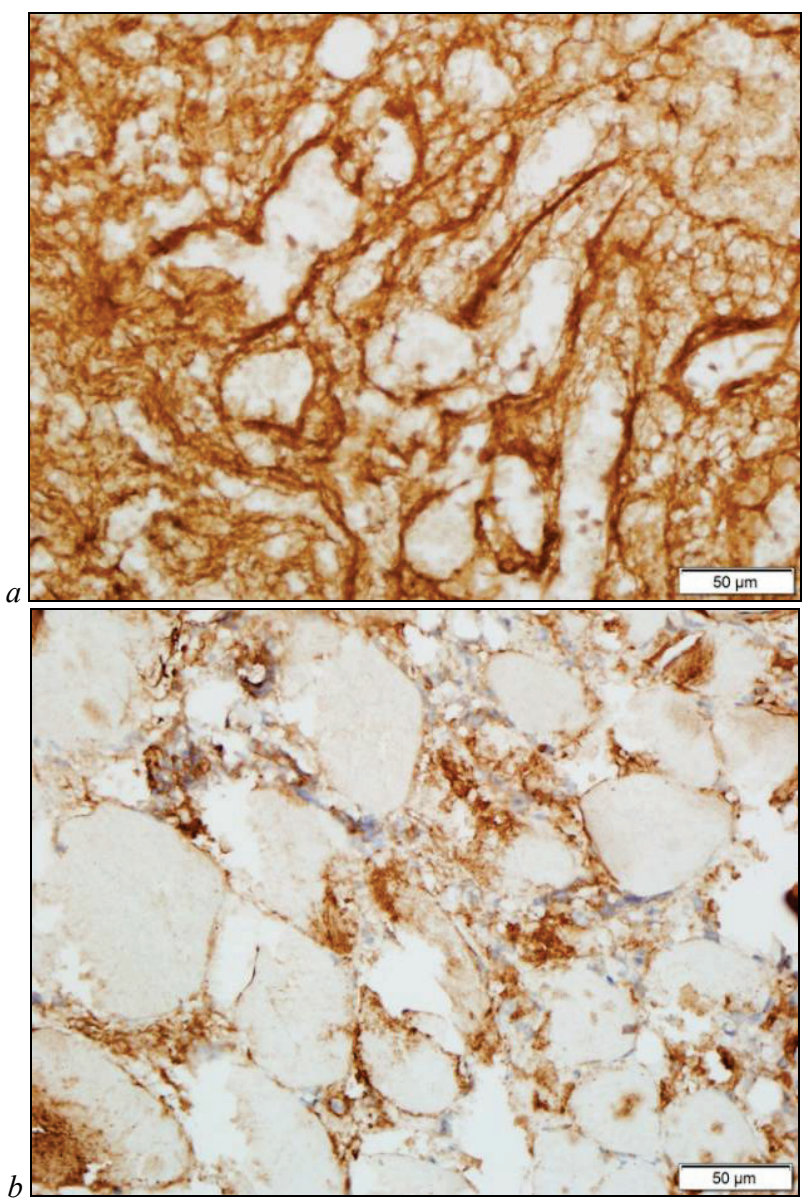

Fig. 10. Positive reaction to VEGF on $100 \%$ of the territory of a fibrin-blood clot $(a)$ and less than $25 \%$ in the muscle tissue $(b)$; 2nd day after the fracture

5 days after the fracture, the change in the structure of the regeneration manifested also in the VEGF. The highest reaction retained in the fibrin, though was the least clear -3 points. Also, the expression of VEGF was observed in endotheliocytes, insignificantly differentiated cells and fibroblasts, which was in general assessed at 2 points.

On the 8 and 12th days after the fracture, the VEGF expression in the regeneration zone was generally moderate or clear (2-3 points). Positive reaction was observed in the regeneration cells - fibroblasts, endotheliocytes, osteoblasts and osteocytes. The matrix of the new formed bone trabeculae was characterized by absence of reaction to VEGF (0 points) (Fig. 11).

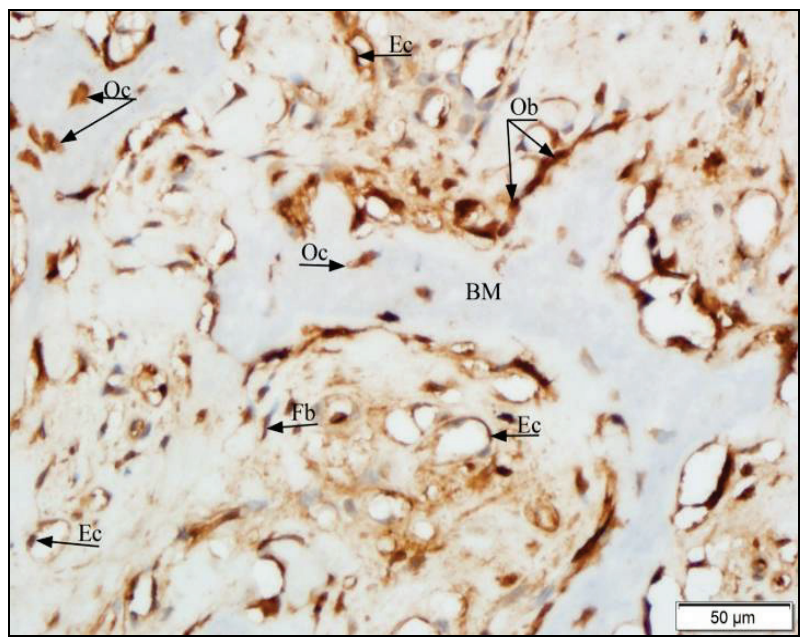

Fig. 11. Reaction to VEGF in the area surrounding the regeneration: the absence of reaction in bone matrix (BM), presence - in the cells; $\mathrm{Oc}$ - osteocyte, $\mathrm{Ob}$ - osteoblast, $\mathrm{Fb}$ - fibroblast, $\mathrm{Ec}$ - endotheliocyte; bar $-20 \mu \mathrm{m}$

Thus, the filling of the fibrin-blood clot around the fragments develops in the direction from the surrounding tissues and lasts 12-18 days among humans. During this period, the cells become osteogenically differentiated. Such tempi and orientation of the process occur due to the presence of fibrin with growth factors' concentration in it.

\section{Discussion}

Traditionally, bone regeneration is divided into the following stages: inflammation (1-7 days), migration and proliferation of cells, formation of the cellular blastema (soft callus, 2-3 weeks), formation of regenerated bone or bone-cartilage (hard callus, 3-4 months), remodeling of the new formed bone tissue ( 5 months and more) (Sfier et al., 2005; Korzh \& Dedukh, 2006; Ito \& Perren, 2007). In our opinion, the inflammatory process is a general reaction of the organism and follows the recovery of any tissue to the end, and the first important stage of bone regeneration at the local level is formation of the fibrin-blood clot, the structure of which stabilizes approximately in 12 to 24 hours (Popsuishapka et al., 2015; Wang et al., 2016).

It should be mentioned that in normal condition, compact bone has a highly organized microstructure in the form of dense lengthwise positioned osteons. Every osteon consists of concentric layers, where the collagen fibers lie in parallel and in a certain direction (Figurskaya, 2007). For the recovery of such structure of the compact bone, the 4-6 months prescribed for treatment are not enough. In the process of bone regeneration after a fracture, first, trabecular tissue forms, then, under the impact of loads, transforms along the periphery to compact bone. Insufficient density of the trabecular bone on the first stages of suppuration in the fracture is compensated by its form and internal structure. Therefore, it was important for us to analyse the mechanisms of formation of trabecular regeneration between the fragments and determine the conditions necessary for this.

As we have seen during an open reduction of the fragments, the bleeding after the bone fracture is caused by two phenomena - saturation of the surrounding tissues with blood and formation of fibrinblood clot from the blood which fills the space around the fracture. There is always a border between the space filled with blood and the surrounding tissues. It is logical to presume that the bleeding from the damaged vessels will last while the blood pressure in the closed cavity around the fracture does not stabilize with the pressure in the vessels. Heightened blood pressure deforms the surrounding tissues as a membrane, therefore causing the spindle-like shape of the hematoma (Litvishko et al., 2016). At first, part of the blood, while in liquid state, penetrates to the surrounding soft tissues. We found accumulations of 
erythrocytes in the muscles, therefore there might have been other elements of blood (thrombocytes, monocytes, etc). At the same time, we observed no formation of fibrin in this zone, despite the fact that fibrogen could be transported here with the blood plasma.

In vitro studies have proved that the structural organization of fibrin-blood clots is affected by mechanical and chemical factors (Popsuishapka et al., 2013; Wang et al., 2016). Among the mechanical factors, the surface pressure is important. It was proved that on the surface of the contact with the trabecular layer which causes pressure, a layer of dense fibrin forms, and in the central part - its structure is cellular (Popsuishapka et al., 2013). A similar structure of fibrin clot was determined in microscopic examination of the biopsy material received from experimental animals (Popsuishapka et al., 2015). This made it possible to represent the general structure of a fibrin-blood clot around a fracture, which occurs after a closed fracture (Fig. 12).

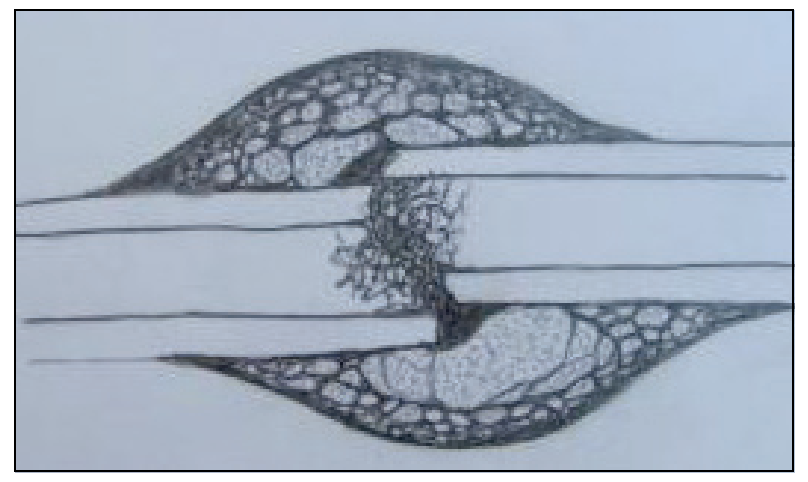

Fig. 12. Schematic image of the structure of a fibrin-blood clot after a closed diaphyseal bone fracture

There is a presumption that a fibrin-blood clot is a "network of closely intertwined fibers of fibrin and blood cells, thrombocytes and blood plasma involved in this network" (https://dic.academic.ru/ dic.nsf/medic2/49113). On the basis of the examinations conducted, we determined that fibrin fibers form membranes of different thickness, positioned at different densities. It could be important that in a threedimensional plane, fibrin membranes form closed cells containing cells of blood serum. The oval shape of the cells indicates the pressure of the fluid inside. During the retraction of fibrin or additional external loads, tension and deformations (including the fact that the fluid does not compress) form in these membranes. They can have a significant effect on the processes of the cell orientation and differentiation.

The process of formation of fibrin takes place first of all due to thrombocytes which are the source of VEGF (Hu \& Olsen, 2016). Using the immunohistological method, we determined the highest reaction to VEGF in the fibrin-blood clot around the fragments, which occurred due to release of growth factors (including VEGF) from the thrombocytes in the process of blood coagulation on the one hand, and mechanical stimuli which increase the content of VEGF, on the other hand (Groothuis et al., 2010). This causes a strong localized signal which activates and orientates the formation of capillaries, which in its turn is a precondition of bone regeneration (Street et al., 2002; Hu \& Olsen, 2016; Ramasamy et al., 2016). The impact of VEGF is determined in time: at the very beginning of the healing, process it concentrates in fibrin and activates neoangeogenesis, later - stimulates the formation of osteoblasts and mineralization of regeneration ( $\mathrm{Hu} \&$ Olsen, 2016; Grosso et al., 2017).

Blood which at first saturated the surrounding vital tissues provides a signal for proliferation of the cells. Their source, as we know, is the cells of the vessels' adventitia, perimysium cells, the cambium layer of the periosteum. Insignificantly differentiated mesenchymal cells come from the side of the adjacent tissues into the formed clot which over a week transforms in cellular blastema along the perimeter. Fibrin carcass functions as a flexible matrix, through which the cells first develop collagen and then bone regeneration.

The mechanisms which orientate the differentiation of cells in an osteogenic direction are still not fully understood. Currently, a com- mon presumption is that the osteogenic differentiation of cells is related to angiogenesis and VEGF (Maes et al., 2010; Hu \& Olsen, 2016; Grosso et al., 2017). We have also observed formation of osteoblasts and osteoid near the blood capillaries. However, at the same time, we found an increased number of vessels also in the young connective tissue. We think that the mechanism of osteogenic differentiation works when the formation of vessels and conditions of oxygenation occur before or coincide in time with the phase of proliferation and differentiation of cells. Perhaps, the high content of VEGF in fibrin over the first 5 days after a fracture causes initial osteogenesis in this particular area.

It is very likely that the osteogenic differentiation of cells is caused also by sufficient level of tension in fibrin-collagen partitions. The elastic properties were proved and the fibrin fiber's elastic modulus was found to equal 1.6-8.0 MPa (Janmey et al., 2009). If the periosteum detached from the ends of the fragments remains, the clot becomes surrounded by a pressurized membrane due to the higher elastic modulus of the periosteum (Evans, 2012), which increases the tension-deformed condition of fibrin (Litvishko et al., 2016). The forces of internal pressure of the fibrin carcass determine the orientation of mechanocytes which form trabeculae of the corresponding form (Claes et al., 1998; Popsuishapka et al., 2015), and, perhaps, initiate the osteogenic differentiation of cells. The more dense (solid) is the partition, the higher is its ability to tense compared to a less dense one in the conditions of similar deformations. The internal forces which developed in it influence the cell cytoplasm and therefore "inform" it about the orientation of the tissue differentiation. The higher the pressure on the cell, the higher the necessity to resist it by formation of a base mechanical substation around to prevent its destruction.

Earlier, we demonstrated that the form and sizes of the formed periosteum bone regeneration after diaphyseal fracture are similar in form and are close in size to the hematomas around the fragments (fibrinblood clot) in sonographic image (Litvishko \& Popsuishapka, 2017).

We can imagine a development of the process when the function of fibrin-formation is decreased, which often occurs in clinical practice at the condition of hemodilution, consumption of aspirin or nonsteroid anti-inflammatory preparations. In this case, blood in liquid state infiltrates the surrounding tissues, becoming remote from the spot where the bone regeneration should take place. In this situation, regeneration occurs in the surrounding tissues through formation of connective tissue (fibrosis).

The described conception involves proving the priority of these phenomena of fibrin-formation in the zone between the fragments after the fracture, which is the initial stage of the fracture healing process. It is wrong to consider the inflammatory stage the beginning of the process. It is not logical at least due to the fact that "inflammation" is a general term and does not indicate a particular phenomenon or factor. Each clinically identified stage of the process should indicate its essence and have practical significance. If the beginning of the fracture healing is inflammation, then why do all doctors inhibit it with all available measures? That is not logical.

Our principles of treatment of fractures are based on the suggested conception, the validity of which is substantiated by clinical results (Litvishko, 2017). They prove that fractures heal faster, and also have a lower frequency of complications if the treatment tactics includes maintaining the integrity of tissues around the fragments with initial formation of a fibrin-blood clot, and also creating a regime of their elastic seaming.

\section{Conclusion}

Formation of a structured fibrin-blood clot around the fragments should be considered the initial stage of the process of formation of bone regeneration after fracture. It is an initial biologically active mechanical carcass for proliferation and osteogenic differentiation of cells. Such conception is a reason for introducing updated principles of fracture treatment, which are related to preserving the surrounding tissues, the formed fibrin-blood clot and creating conditions which provide a manageable regime of regeneration tensions. 


\section{References}

Antonova, E., Le, T. K., Burge, R., \& Mershon, J. (2013). Tibia shaft fractures: Costly burden of nonunions. BMC Musculoskeletal Disorders, 14(1), 42.

Aydemir Turkal, H., Demirer, S., Dolgun, A., \& Keceli, H. G. (2016). Evaluation of the adjunctive effect of platelet-rich fibrin to enamel matrix derivative in the treatment of intrabony defects. Six-month results of a randomized, split-mouth, controlled clinical study. Journal of Clinical Periodontology, 43(11), 955-964.

Bastami, F., \& Khojasteh, A. (2016). Use of leukocyte-and platelet-rich fibrin for bone regeneration: A systematic review. Regeneration, Reconstruction and Restoration, 1(2), 47-68.

Claes, L. E., Heigele, C. A., Neidlinger-Wilke, C., Kaspar, D., Seidl, W., Margevicius, K. J., \& Augat, P. (1998). Effects of mechanical factors on the fracture healing process. Clinical Orthopaedics and Related Research, 355S, S132-S147.

Dohan Ehrenfest, D. M., Del Corso, M., Diss, A., Mouhyi, J., \& Charrier, J. (2010). Three-dimensional architecture and cell composition of a choukroun's platelet-rich fibrin clot and membrane. Journal of Periodontology, 81(4), 546-555.

Dong, L., Yin, H., Wang, C., \& Hu, W. (2014). Effect of the timing of surgery on the fracture healing process and the expression levels of vascular endothelial growth factor and bone morphogenetic protein-2. Experimental and Therapeutic Medicine, 8(2), 595-599.

Echeverri, L. F., Herrero, M. A., Lopez, J. M., \& Oleaga, G. (2015). Early stages of bone fracture healing: Formation of a fibrin - collagen scaffold in the fracture hematoma. Bulletin of Mathematical Biology, 77(1), 156-183.

Maes, C., Kobayashi, T., Selig, M. K., Torrekens, S., Roth, S. I., Mackem, S. \& Kronenberg, H. M. (2010). Osteoblast precursors, but not mature osteoblasts, move into developing and fractured bones along with invading blood vessels. Developmental Cell, 19(2), 329-344.

Marsell, R., \& Einhorn, T. A. (2011). The biology of fracture healing. Injury, $42(6), 551-555$

McNally, E. G. (2011). The development and clinical applications of musculoskelet alultrasound. Skeletal Radiology, 40(9), 1223-1231.

Moreno-Miralles, I., Schisler, J. C., \& Patterson, C. (2009). New insights into bone morphogenetic protein signaling: Focus on angiogenesis. Current Opinion in Hematology, 16(3), 195-201.
Popsuishapka, A. K., Lytvyshko, V. A., \& Ashukina, N. A. (2015). Klinikomorfolohichni stadiyi zroshchennya vidlamkiv pislya perelomu kistky [Clinical and morphological stages of bone fragments fusion]. Orthopaedics, Traumatology and Prosthetics, 1, 12-20 (in Ukrainian).

Popsuishapka, A. K., Lytvyshko, V. A., Ashukina, N. A., \& Danyshchuk, Z. N. (2013). Osobennosti formirovaniya, strukturno-mekhanicheskiye svoystva fibrin-krovyanogo sgustka i yego znacheniye dlya regeneratsii kosti [Peculiarities in the formation, structural-mechanical properties of a fibrin-blood clot and its importance for bone regeneration in fractures]. Orthopaedics, Traumatology and Prosthetics, 4, 5-12 (in Russian).

Raica, M., \& Cimpean, A. M. (2010). Platelet-derived growth factor (PDGF)/PDGF receptors (PDGFR) axis as target for antitumor and antiangiogenic therapy. Pharmaceuticals, 3(12), 572-599.

Ramasamy, S. K., Kusumbe, A. P., Schiller, M., Zeuschner, D., Bixel, M. G., Milia, C., \& Adams, R. H. (2016). Blood flow controls bone vascular function and osteogenesis. Nature Communications, 7, 13601

Schell, H., Duda, G. N., Peters, A., Tsitsilonis, S., Johnson, K. A., \& SchmidtBleek, K. (2017). The haematoma and its role in bone healing. Journal of Experimental Orthopaedics, 4(1), 5.

Sfeir, C., Ho, L., Doll, B. A., Azari, K., \& Hollinger, J. O. (2005). Fracture repair. In: Bone regeneration and repair: Biology and clinical applications. Humana Press. Pp. 21-44.

Sivaraj, K. K., \& Adams, R. H. (2016). Blood vessel formation and function in bone. Development, 143(15), 2706-2715.

Street, J., Bao, M., de Guzman, L., Bunting, S., Peale, F. V., Ferrara, N., Steinmetz, H., Hoeffel, J., Cleland, J. L., Daugherty A., van Bruggen, N., Redmond, H. P., Carano, R. A. D., \& Filvaroff, E. H. (2002). Vascular endothelial growth factor stimulates bone repair by promoting angiogenesis and bone turnover. Proceedings of the National Academy of Sciences of the United States of America, 99(15), 9656-9661.

van Meeteren, L. A., Goumans, M., \& Ten Dijke, P. (2011). TGF- $\beta$ receptor signaling pathways in angiogenesis: Emerging targets for anti-angiogenesis therapy. Current Pharmaceutical Biotechnology, 12(12), 2108-2120.

Wang, X., Friiis, T. E., Masci, P. P., Crawford, R. W., Liao, W. \&Xiao, Y. (2016) Alteration of blood clot structures by interleukin-1 beta in association with bone defects healing. Scientific Reports, 6, 35645 . 